\title{
Extending a System for Measuring Dynamic Knowledge: Reconsidering Knowledge Flow Efficiency for Decision Support
}

\author{
Mark E Nissen \\ US Naval Postgraduate School \\ MNissen@nps.edu
}

\begin{abstract}
Knowledge is key to competitive advantage, but it is inherently invisible, intangible and resistant to quantification, particularly when in dynamic motion. Recent research builds upon emerging knowledge measurement techniques and well-established knowledge flow theory to develop a system for measuring dynamic knowledge in the organization. Results from application to archetypical organization processes are highly consistent with extant theory, but they also lead us to question some theoretic concepts and correspondences. In this article, we extend the measurement system to specify the effects of knowledge flow efficiency. This establishes a novel decision support capability and extends an exciting new line of knowledge management research.
\end{abstract}

\section{Introduction}

Knowledge is key to competitive advantage [3,7,23]: Knowledge enables effective action; effective action drives superior performance; and superior performance supports competitive advantage [13]. Indeed, some scholars argue that knowledge represents the only sustainable source of competitive advantage [5].

However, knowledge does not represent a single, monolithic concept: different kinds of knowledge (e.g., tacit, explicit, individual, group, created, applied) have qualitatively different properties and behaviors, and hence affect action, performance and competitive advantage differently [11]. Neither can knowledge remain static in support of competitive advantage: it must move or flow rapidly and reliably from where and when it is to where and when it is needed in the organization.

This places particular importance on understanding the dynamics of knowledge as it flows, but unfortunately, knowledge is inherently intangible, invisible and resistant to quantification [1], particularly when in dynamic motion. This makes it a considerable and persistent challenge to understand, visualize and measure.

Recent research builds upon emerging knowledge measurement techniques and well-established knowledge flow theory to develop a system for measuring dynamic knowledge in the organization [14]. Results from application to archetypical organization processes are highly consistent with extant theory. For instance, measured differences between the dynamics of tacit and explicit knowledge mirror theoretic predictions closely. However, they also lead us to question some longstanding theoretic concepts and correspondences. For instance, the concept knowledge flow efficiency exhibits difficulties when instantiated via dynamic knowledge measurements.

In this article, we extend our system for measuring dynamic knowledge in the organization to specify the effects of knowledge flow efficiency. This establishes a novel decision support capability. It also extends an exciting new line of knowledge management (KM) research that further enhances our ability to visualize and measure dynamic knowledge.

\section{Background}

After casting a wide metaphoric net in terms of relevant literatures to review (e.g., Economics, Education, Information Theory, Knowledge Management) for background, insight and inspiration [14], the research noted above draws judiciously and analogically from our understanding of dynamic physical systems to conceptualize a set of equations for measuring dynamic knowledge.

As a fundamental cognitive process [18], employed by adults [24] and children [25] alike, analogic reasoning represents a notably powerful learning and communication approach that spans many domains, including Design [2], Organization [27], Physics [19], Strategy [6], Supply Chain [9], and many others. Analogies can promote creativity, in both people and computers [8], and they can 
facilitate thinking in domains with negligible precedent, such as Outer Space Law [17].

In this section we provide an overview of dynamic physical equations used to guide our analogic reasoning. We then conceptualize and specify an analogic set of dynamic knowledge equations.

\subsection{Dynamic physical equations}

To recapitulate the approach, which is described in detail through the research noted above [14], a simple physical system is represented mathematically through the basic Newtonian equations summarized in Table 1. Such equations can be found in any introductory Physics textbook, yet they enable quantitative measurement, analysis, prediction and simulation of dynamic physical systems. Here we interrelate force (mass $\mathrm{x}$ acceleration; expressed in Newtons), work (force $\mathrm{x}$ distance; expressed in Joules) and power (work / time; expressed in Watts).

Table 1 Physical system equations

\begin{tabular}{|l|l|l|}
\hline Construct & \multicolumn{1}{|c|}{ Description } & Equation \\
\hline Force (F) & $\begin{array}{l}\text { Effort required to } \\
\text { accelerate mass }\end{array}$ & (1) F = m x a \\
\hline Work (W) & $\begin{array}{l}\text { Force applied } \\
\text { through distance }\end{array}$ & (2) W = F x d \\
\hline Power (P) & $\begin{array}{l}\text { Work done per unit } \\
\text { time }\end{array}$ & (3) P = W / t \\
\hline
\end{tabular}

We note also (beyond the table) how work and energy are exchangeable and expressed in the same units (Joules): energy is required to perform work, and work performance involves the expenditure of energy. We leverage such exchangeability below through analogic reasoning for knowledge systems.

We note further how friction affects many physical systems by opposing motion and acceleration. An ordinary shopping cart, for instance, requires greater effort (i.e., more force) to push down a store aisle with a rough floor than a smooth one: the greater friction associated with the rough floor opposes motion and acceleration of the cart, hence it requires more force to push.

Considering friction in support of our analogic reasoning, a simple, linear, negative relationship between force-including that required to overcome friction $\left(\mathrm{F}_{\mathrm{Fr}}\right)$ - and floor smoothness (fs) is delineated in Figure 1. Here force can be measured in Newtons, and smoothness is expressed on a $[0,1]$ continuum between rough ( $f s=0$ ) and smooth ( $f s=1$ ) endpoints.

Specifically as depicted in the figure, a rough floor is characterized here as requiring ten times the force to push a shopping cart as that needed on a smooth floor $\left(\mathrm{F}_{\mathrm{Fr}}=10\right.$ - 9fs). This downward sloping relationship between force and smoothness is representative, with specific slopes, intercepts and functions highly likely to differ across various carts, stores, aisles and floors. Nonetheless, the relationship makes intuitive sense and is consistent with many physical observations and measurements.

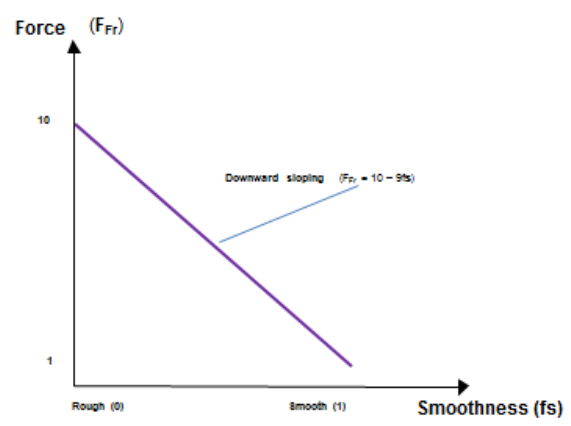

Figure 1 Force and smoothness

\subsection{Dynamic knowledge equations}

As summarized in Table 2, we outline an analogic system for measuring dynamic knowledge. The specifications and analogic mappings are detailed through prior research [14], so we present only concise summaries in this section. To recapitulate a key point, however, none of these analogic constructs or relationships is intended to be precise or perfect. Rather, they are intended to elucidate the dynamics of knowledge and to help us compose a simple, novel and insightful system for measurement.

Table 2 Analogic knowledge system

\begin{tabular}{|l|l|l|}
\hline Construct & \multicolumn{1}{|c|}{ Description } & Analogy \\
\hline $\begin{array}{l}\text { K-Force } \\
\text { (KF) }\end{array}$ & $\begin{array}{l}\text { Effort required to } \\
\text { accelerate knowledge }\end{array}$ & $\mathrm{f}(\mathrm{C}, \mathrm{E}, \mathbf{0})$ \\
\hline $\begin{array}{l}\text { K-Work } \\
\text { (KW) }\end{array}$ & $\begin{array}{l}\text { K-Force applied } \\
\text { through Reach }\end{array}$ & KF x R \\
\hline $\begin{array}{l}\text { K-Power } \\
\text { (KP) }\end{array}$ & $\begin{array}{l}\text { K-Work done per unit } \\
\text { flow time }\end{array}$ & KW / FT \\
\hline
\end{tabular}

Briefly, knowledge force (KF) is analogous to physical force and represents the effort required to accelerate knowledge in an organization. From Knowledge Flow Theory (KFT; [4,7,12,16,21,23]), it is expressed as a function of the knowledge chunks (C) [22] being accelerated and the explicitness (E) of 
such knowledge. In this conceptualization, one chunk of knowledge can enable the performance of one atomic action in the organization.

Explicitness derives from Nonaka's [16] epistemological dimension and represents the degree to which a knowledge chunk has been articulated in explicit form. Reading a book about how to fly an airplane, for instance, would represent purely explicit knowledge, whereas a person's experience flying an airplane would represent purely tacit knowledge.

The greater the number of chunks being accelerated (analogous to physical mass), and the more tacit the corresponding knowledge (analogous to physical friction), the greater the K-Force required. Notice also the o vector representing a number of other, unspecified factors (e.g., experience, communication skill, motivation, stress, organization climate, IT support), which are likely to play a role, but which have yet to be integrated explicitly or analogically.

Reach (R) derives from Nonaka's [16] ontological dimension and represents the number of people able to utilize the knowledge chunks from above (analogous to physical distance). Reach combines with K-Force to specify knowledge work (KW) accomplished in the organization (analogous to physical work). Parallel to the exchange between and common units of work and energy in physical systems, we also conceptualize a correspondence between knowledge work and knowledge energy (KE): K-Energy is required to perform K-Work, and $\mathrm{K}$-Work performance involves the expenditure of $\mathrm{K}$ Energy.

In turn, flow time (FT) represents the time required for such knowledge chunks to flow from one person (e.g., an expert), group (e.g., a sales team), place (e.g., West Coast office) or time (e.g., night shift) to another. As a temporal measure, it combines with $\mathrm{KW}$ to specify knowledge power (KP), which represents the knowledge work accomplished (and knowledge energy expended) per unit time (analogous to physical power).

Continuing to draw analogically from the dynamics of physical systems, and considering friction, which opposes motion and acceleration, a simple, linear, negative relationship between knowledge force (KF) and explicitness (E) is included as Equation (4) in Table 3. Consistent with KFT, this relationship indicates that tacit knowledge, which is notably "sticky" [26] and difficult to move through the organization, requires more effort (i.e., greater $\mathrm{KF}$ ) to accelerate than its explicit counterpart.

Indeed, parallel to the representative physical interrelation between force, friction and floor smoothness described above (i.e., $\mathrm{F}_{\mathrm{Fr}}=10$ - 9fs), a chunk of tacit knowledge is characterized here as requiring ten times $(10 \mathrm{x})$ the $\mathrm{K}$-Force as that needed to get a chunk of explicit knowledge flowing. We refer to units of K-Force as "Nonakas" (N), acknowledging the seminal knowledge flow research done by Nonaka [16].

Table 3 Knowledge system equations

\begin{tabular}{|l|l|}
\hline Construct & \multicolumn{1}{|c|}{ Equation } \\
\hline K-Force & (4) KF $=\mathrm{C} \times(10-9 \mathrm{E}) \times \mathbf{~ o}$ \\
\hline K-Work & (5) KW = KF x R (= KE) \\
\hline K-Power & (6) KP = KW / FT \\
\hline
\end{tabular}

K-Work (and K-Energy) then follows in Equation (5) as the product of K-Force and Reach (R). We refer to units of K-Work (and K-Energy) as "Polanyis" (P), for the keen insight into tacit knowledge provided by Polanyi [20]. K-Power is specified in turn through Equation (6) by dividing KWork (or K-Energy) by flow time (FT). We refer to K-Power as "Bacons" (B), acknowledging Sir Francis Bacon, to whom many scholars attribute the aphorism, "knowledge is power." The interested reader can refer to [14] for details.

\section{Archetypical Application}

In this section we apply the set of knowledge equations developed above to contrasting knowledge flow archetypes from the literature. We begin by outlining a multidimensional approach to visualizing dynamic knowledge, which utilizes many of the measurement constructs described above. Then we apply the corresponding measurement system directly.

\subsection{Dynamic knowledge visualization}

To outline this multidimensional visualization approach, which is described in detail through prior research [14], we refer to Figure 2. The vertical axis represents explicitness, which is one of the knowledge measurement constructs from above and derives from Nonaka [16]. The horizontal axis represents reach, which is another of the knowledge measurement constructs from above and derives from Nonaka also. The third axis represents life cycle, which is helpful for visualization and used to extend Nonaka's model [10]. Life cycle pertains to what is being done with knowledge (e.g., create, share, apply).

Flow time is not delineated via separate axis, but it is another of the knowledge measurement constructs from above and used to extend Nonaka's 
model further. Within the context of this multidimensional visualization scheme, flow time represents the time required for knowledge to flow between any two coordinate points in the space (e.g., Points A and B in the figure). When knowledge flows quickly through an organization (i.e., when flow time is short), for instance, we delineate the corresponding flow with a relatively thin vector arrow, whereas a comparatively thick one is used when knowledge flows slowly. Our expectations from KFT are that tacit knowledge, which is notably "sticky" and difficult to move through the organization, will flow more slowly than its explicit counterpart. Hence tacit flows would generally be represented by relatively thick arrows, whereas comparatively thin ones reflect explicit flows better.

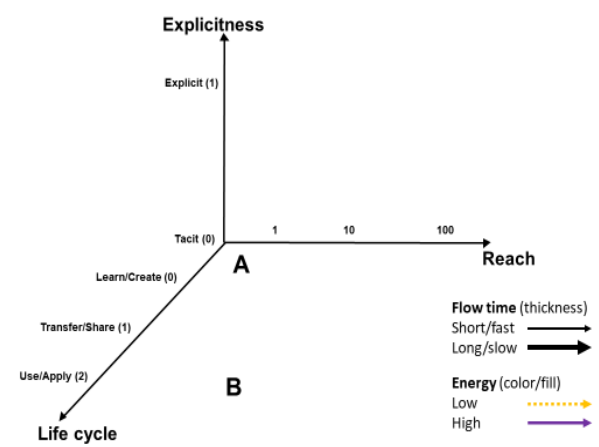

\section{Figure 2 Knowledge visualization space}

Finally, we also utilize different vector arrows to delineate knowledge energy, which is noted above with correspondence to the measurement construct knowledge work, and which represents the performance level of actions enabled by knowledge as it flows through the organization. Higher energy knowledge flows (e.g., that enable higher performance levels of knowledge work) are delineated with solid (purple) vector arrows, for instance, whereas dotted (orange) arrows are used for lower energy knowledge. Our expectations from KFT are that tacit knowledge, which can enable higher performance levels, will flow with greater energy than its explicit counterpart. Hence tacit flows would generally be represented by solid (purple) arrows, whereas dotted (orange) ones reflect explicit flows better. In theory, flow time and knowledge energy represent orthogonal dimensions, but in practice, they may covary.

In terms of measurement, explicitness can be represented as a continuous dimension, with tacit and explicit endpoints on a ratio scale $[0,1]$. This implies that various combinations of tacit and explicit streams may comprise some knowledge flows. Such conceptualization as a continuous dimension also serves to extend much prior research (e.g., [16]), which views tacit and explicit knowledge more as a categorical contrast than a continuum.

Reach can be measured along an integer scale (e.g., 1, 10, 100), enumerating the number of people who can utilize knowledge. Life cycle represents an iterative sequence of activities, with a somewhat arbitrary ordinal scale (e.g., $0,1,2)$ referring to what is being done with knowledge. Flow time can be measured along a ratio scale using a stopwatch, calendar, employee timecard, or like instrument. As noted above, $K$-Energy (and $K$-Work) is calculated as the product of K-Force and Reach.

Together, this multidimensional framework enables the visualization of dynamic knowledge and is very general. Theoretically, any dynamic flow of knowledge can be characterized in terms of these dimensions and delineated in this space, and in theory, knowledge can flow via an infinite number of different paths between any two points.

Consider, for example, Points A and B in Figure 2. Say that an individual worker in the organization discovers some new and useful knowledge (Point A), and management is interested in having all ten people in a group learn and apply such knowledge (Point B). Say further that the knowledge is tacit and represents 100 chunks. This implies that the individual worker could perform 100 novel atomic actions (or one novel compound action comprised of 100 atomic elements, or some conforming combination of atomic and compound actions). There are clearly many different organization sharing processes available to enable this new knowledge to flow between the individual and his or her group members, hence equally many corresponding knowledge flow paths through the multidimensional space are possible too.

Figure 3 delineates two, contrasting, archetypical knowledge flows. In the flow labeled "Explicit Path," say that the individual worker (Point A) expends time and energy to articulate his or her knowledge in explicit form (e.g., written instructions, graphic depictions, mathematic formulae and calculations, solved examples). This is represented by Point $\mathrm{M}$ in the figure. Then this individual could encode such explicit knowledge digitally within a computer network (e.g., via email attachment, website resource, document repository), which could be shared very quickly with all ten coworkers, wherever in the world they happen to be located. This is represented by Point $\mathrm{N}$ in the figure.

After sharing as such, each of the coworkers could apply the knowledge directly to his or her work activities (Point B). This organization process and 
corresponding knowledge flow path are illustrated by light (orange) dotted vector arrows in the figure to represent the explicit nature of the dynamic knowledge. The first segment (i.e., A-M) is delineated with a relatively thick vector to indicate that the process of articulating tacit knowledge into explicit form can be time consuming, particularly when compared to the other segments corresponding to explicit knowledge sharing (i.e., $\mathrm{M}-\mathrm{N}$ ) and application (i.e., N-B). By using a stopwatch, calendar, employee timecard, or like instrument, researchers or managers could measure the time required for this knowledge to flow from $\mathrm{A}$ to $\mathrm{B}$, and hence obtain a measured value for flow time.

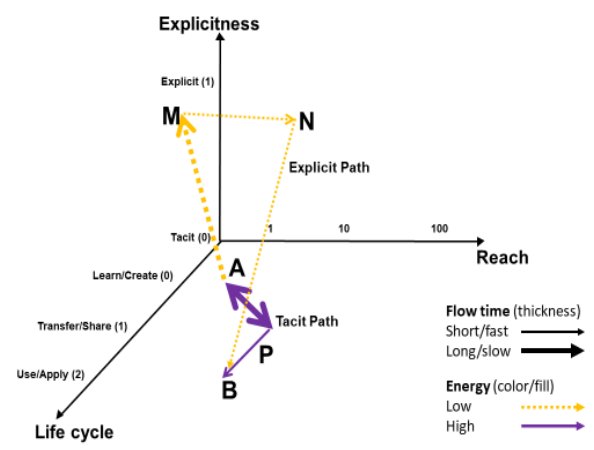

Figure 3 Knowledge flow archetypes

In the flow labeled "Tacit Path," say that the individual worker interacts interpersonally with the group members, working closely with these people, soliciting and answering their questions, observing and correcting the coworkers as they practice, and both mentoring and coaching them until everyone in the group has learned the knowledge. This is represented by Point $\mathrm{P}$ in the figure.

With such learning accomplished effectively, all ten coworkers would be able to apply the knowledge directly to their work activities (Point B). This Tacit Path differs greatly from the Explicit Path above, and the corresponding knowledge flow is illustrated by dark (purple) solid vector arrows in the figure to represent the tacit nature of the dynamic knowledge.

The first segment (i.e., A-P) is delineated with a relatively thick arrow to indicate that the process of sharing tacit knowledge can be especially time consuming, particularly when compared to the other segment corresponding to tacit knowledge application (i.e., P-B). This first segment is delineated with a double headed arrow also to indicate that knowledge sharing goes both ways: the individual worker (Point A) is learning (e.g., group norms) from the other members as they interact interpersonally, and the coworkers are learning (esp. the new knowledge) from this individual.

As above, researchers or managers could use the same stopwatch, calendar, employee timecard, or like instrument to measure the time required for knowledge to flow from $\mathrm{A}$ to $\mathrm{B}$, and hence obtain a measured value for flow time along this alternate, tacit path. Since these two, contrasting, archetypical knowledge flow paths are very different, one would expect for the corresponding flow times and energy levels to differ accordingly.

\subsection{Dynamic knowledge measurement}

Table 4 summarizes three key measured values for each archetypical knowledge flow. The rationale and technique for each measurement are described in detail through prior research [14], so we present only summary results here. For the 100 chunks moving through the Explicit Path, K-Energy (KE) totals 2550 Polanyis. (KE and FT values are summarized as thousands in the table.) Worker timecards are used to measure flow time (FT) of nearly five hours $(16,100$ seconds) for the flow, which combines to reveal the K-Power (KP) measurement of 0.16 Bacons.

For the same 100 chunks moving through the Tacit Path, K-Energy totals 20,000 Polanyis with flow time over 15 hours (55,100 seconds), which combines to reveal the K-Power measurement of 0.36 Bacons.

Table 4 Knowledge flow path comparison

\begin{tabular}{|l|c|c|c|l|}
\hline Path & \multicolumn{1}{|c|}{ KE } & \multicolumn{1}{|c|}{ FT } & \multicolumn{1}{|c|}{ KP } & Comment \\
\hline Explicit & 2.55 & 16.1 & 0.16 & $\begin{array}{l}\text { Less energy } \\
\text { Less time }\end{array}$ \\
\hline Tacit & 20.00 & 55.1 & 0.36 & $\begin{array}{l}\text { More energy } \\
\text { More time }\end{array}$ \\
\hline Ratio & 7.8 & 3.4 & 2.3 & "Best"? \\
\hline
\end{tabular}

Notice that the tacit knowledge flows at nearly eight times (7.8) the energy (and accomplishes comparably greater K-Work) of its explicit counterpart (20,000 vs. 2550 P), but it takes more than three times (3.4) as long to flow (55,100 vs. $16,100 \mathrm{~s})$. Which archetype is "best" depends upon the circumstances: Where knowledge is required to flow quickly, and the organization can tolerate the lower energy level (i.e., performance level) corresponding to the Explicit Path, the first archetype would be preferable. Alternatively, where the performance level (i.e., energy level) must be high, and the organization can wait for tacit knowledge to flow, the Tacit Path would be preferable. These measurements are highly consistent with KFT [14]. 


\section{Model Extension}

In this section we extend our system of knowledge equations to increase fidelity and enhance its capability for analysis and comparison across organization knowledge flows. We begin by conceptualizing knowledge flow efficiency through continued analogic reasoning. Such conceptualization is followed in turn by graphic and numeric application, through which we employ the extended system of knowledge equations to visualize, measure and compare the well-known Spiral Model [16] with the two archetypical knowledge flow patterns from above.

\subsection{Knowledge flow efficiency}

Recall from above the correspondence between K-Work and K-Energy (i.e., KW = KE). This correspondence assumes that the amount of work accomplished through a particular knowledge flow equals the energy associated with such flow. Reconsidering the physical system supporting our analogic reasoning, this would imply perfect efficiency, meaning that all energy expended by a system is converted to useful work.

Efficiency of physical systems (e.g., heat engines) is expressed often as the ratio of work accomplished to energy expended (e.g., $E_{P}=W / E$, where $E_{P}$ represents efficiency of the physical system, W represents work accomplished, and E represents energy expended). For a physical system with perfect efficiency (i.e., $E_{P}=1$ ), work and energy would be equal.

Nearly every physical system suffers from energy losses (e.g., from thermal radiation), however, meaning that the amount of work accomplished by a physical system is generally less than the amount of energy expended by it. Hence the perfectly efficient physical system is unlikely in practice (i.e., $\mathrm{E}_{\mathrm{P}} \leq 1$ ).

Analogously our implicit equivalence between KWork and K-Energy is unlikely in practice also, and nearly every knowledge system probably suffers from energy losses too (e.g., from o vector factors). As expressed in Equation (7a), we extend the system of dynamic knowledge equations to specify knowledge flow efficiency $\left(\mathrm{E}_{\mathrm{K}}\right)$ as the ratio of knowledge work accomplished (KW) relative to knowledge energy expended (KE).

Equation (7a) $\mathrm{E}_{\mathrm{K}}=\mathrm{KW} / \mathrm{KE}$

Rearranging the terms a bit, we express K-Work as a function of K-Energy in Equation (7b).
Equation (7b) KW = KE x $\mathrm{E}_{\mathrm{K}}$

Now substituting Equation (5) from above for KEnergy (i.e., KF x R = KE), we derive Equation (7c) for K-Work.

Equation (7c) $\mathrm{KW}=\mathrm{KF} \times \mathrm{R} \times \mathrm{E}_{\mathrm{K}}$

Clearly where $E_{K}$ equals one, K-Work and KEnergy are equivalent as in Equation (5) above, but for all (likely) efficiency values below that (i.e., $\mathrm{E}_{\mathrm{K}} \leq$ 1 ), some energy loss $\left(E_{L}\right)$ is expected. Such loss is expressed in Equation (7d).

Equation (7d) $\mathrm{E}_{\mathrm{L}}=\mathrm{KE}-\mathrm{KW}$

To summarize, here we extend the system of dynamic knowledge equations to incorporate knowledge flow efficiency $\left(E_{K}\right)$ through continued analogy with dynamic physical systems, nearly all of which suffer energy losses. This enables us to differentiate between K-Work and K-Energy (KW = $\mathrm{KE} \times \mathrm{E}_{\mathrm{K}}$ ), to refine the specification of K-Work in terms of K-Force and Reach (KW $=\mathrm{KF} \times \mathrm{R} \times \mathrm{E}_{\mathrm{K}}$ ), and to specify energy loss $\left(\mathrm{E}_{\mathrm{L}}=\mathrm{KE}-\mathrm{KW}\right)$ in terms of knowledge work and energy. These refinements to our system of dynamic knowledge equations should increase fidelity and enhance its capability for analysis and comparison across organization knowledge flows.

\subsection{Spiral Model Visualization}

We continue in this section through visualization of the well-known Spiral Model from the literature [16]. This sets the metaphoric stage for measurement and comparison with the two knowledge flow archetypes from above. We begin with a brief review of knowledge spiral basics, and as with the archetypical flow paths delineated above, we instantiate the associated theoretic model through both multidimensional knowledge visualization and dynamic measurement.

Briefly, the knowledge spiral conceptualizes organization knowledge flowing through iterative conversions (i.e., socialization, externalization, combination, internalization). Each conversion involves tacit and $\backslash$ or explicit knowledge.

Socialization is a tacit-to-tacit flow, as an individual learns experientially from others, for instance. This is similar in many respects to how the Tacit Path from above begins, where knowledge is shared between members of a group. Externalization is a tacit-to-explicit flow, as individual knowledge is 
articulated in explicit form, for instance. This is similar in many respects to how the Explicit Path from above begins, where knowledge rises up from the tacit plane as it is made explicit.

Combination is an explicit-to-explicit flow, as one individual's explicit knowledge is combined with others', for instance. This resembles in many respects how knowledge flows across the Reach dimension in the Explicit Path from above. Internalization is an explicit-to-tacit flow, as knowledge is learned and applied in the organization, for instance. This resembles in many respects how explicit knowledge is applied in the Explicit Path from above. The cycle can continue, as knowledge may spiral out ever further in terms of organization reach, for instance, through a process termed amplification.

As with the archetypical knowledge flow paths delineated above, we begin by representing the knowledge spiral via multidimensional space in Figure 4. To avoid cluttering the figure, we illustrate only one loop of the spiral.

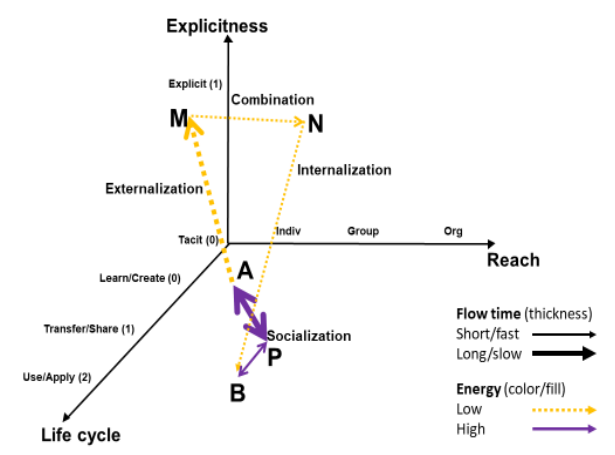

Figure 4 Knowledge spiral representation

Following the description above, we begin the knowledge spiral with socialization, which involves both direct experience and interpersonal interaction [16:19]. The experiential component is represented by a solid (purple) bidirectional arrow between Points $\mathrm{B}$ and $\mathrm{P}$. This represents people in a group working, learning and sharing experiences together. With the thin vector arrow, from KFT, we assume here that most people in the group are experienced and competent, enabling relatively quick application of their knowledge to perform organization work. With less experience and longer flow times, we would delineate such knowledge flow with a thicker arrow. The arrow is bidirectional to indicate knowledge flows for both work performance $(\mathrm{P} \rightarrow \mathrm{B})$ and experiential sharing $(\mathrm{B} \rightarrow \mathrm{P})$.

The interactional component is represented by a solid (purple) bidirectional arrow between Points $\mathrm{P}$ and $\mathrm{A}$. This represents people in a group interacting together with an individual at Point A. With the thick vector arrow, we assume here the individual to be comparatively new to the group and gaining tacit knowledge through socialization. From KFT, this process is likely to be comparatively slow, hence the relatively thick knowledge flow arrow. The arrow is bidirectional to indicate that the individual learns from the group $(\mathrm{P} \rightarrow \mathrm{A})$, and vice versa $(\mathrm{A} \rightarrow \mathrm{P})$. Socialization involves only tacit knowledge, hence the flows are all within the tacit plane of the figure.

The spiral continues with externalization, which involves articulation of tacit knowledge into explicit form. We illustrate such articulation through a dotted (orange) unidirectional arrow between Points $\mathrm{A}$ and M. This represents an individual at Point A converting his or her tacit knowledge into explicit form (e.g., via written document). From KFT, the externalization process is likely to be relatively slow, hence the thick arrow, as considerable time and effort are required often to articulate one's knowledge explicitly.

Combination follows with another dotted (orange) unidirectional arrow, here between Points $\mathrm{M}$ and $\mathrm{N}$. This represents the combination of extant explicit knowledge of different people, shown in the figure as belonging to a group. A relatively thin arrow is used to delineate this combination flow, from KFT, as the process would likely occur comparatively quickly with respect to socialization and externalization, particularly because the extant knowledge has been articulated into explicit form already.

Finally, internalization completes the loop with a dotted (orange) unidirectional arrow between Points $\mathrm{N}$ and B. This represents group learning and application of the knowledge combined from above, which we delineate with a relatively thin arrow, from KFT, to suggest that explicit knowledge flows comparatively quickly.

From here the cycle can continue between individuals in this same group, for instance, socializing, externalizing, combining and internalizing additional knowledge; or it can expand out to the organization level, for instance, as members of the group interact with people from different groups across the organization.

Clearly other interpretations of the knowledge spiral and their corresponding representations via multidimensional space are possible, but this illustrates at least one way in which spiraling knowledge can be delineated and visualized as above. Table 5 summarizes Spiral Model measurements for comparison with the archetypes above. As before, the rationale and technique for each measurement are described in detail through prior research [15], so we 
present only summary results here. Notice that Spiral Model knowledge flows with the most energy (22,550 P) and takes the most time (71,500 s).

Table 5 Spiral flow path comparison

\begin{tabular}{|l|c|c|c|l|}
\hline Path & KE & FT & KP & Comment \\
\hline Explicit & 2.55 & 16.1 & 0.16 & $\begin{array}{l}\text { Less energy } \\
\text { Less time }\end{array}$ \\
\hline Tacit & 20.00 & 55.1 & 0.36 & $\begin{array}{l}\text { More energy } \\
\text { More time }\end{array}$ \\
\hline Spiral & 22.55 & 71.5 & 0.32 & $\begin{array}{l}\text { Most energy } \\
\text { Most time }\end{array}$ \\
\hline
\end{tabular}

\subsection{Model Application}

We continue in this section through application of the extended system of dynamic knowledge equations. This enables direct, numeric comparison of knowledge flows. We begin by comparing the three knowledge flow patterns from above (i.e., Explicit Path, Tacit Path, Spiral Model) as ideal processes that reflect perfect knowledge flow efficiency (i.e., $\mathrm{E}_{\mathrm{K}}=1.0$ ). Although unrealistic in practice, this establishes a baseline for comparison with more practical efficiency levels.

Following the same logic and procedure described for the archetypical paths above, and reflecting the same 100 chunks of knowledge and group size of 10, measured values for the Explicit Path, Tacit Path and Spiral Model are summarized in Table 6. As above, the spiral knowledge flows with more energy (22,550 P) than either its explicit or tacit counterpart. Clearly with perfect knowledge flow efficiency, K-Energy (KE) and K-Work (KW) values are identical, and energy loss $\left(E_{L}\right)$ is zero, across all three knowledge flow patterns.

Table 6 Measurements - Ideal

\begin{tabular}{|l|c|r|r|c|}
\hline Path & $\mathbf{E}_{\mathbf{K}}$ & \multicolumn{1}{|c|}{$\mathbf{K E}$} & \multicolumn{1}{|c|}{$\mathbf{K W}$} & $\mathbf{E}_{\mathbf{L}}$ \\
\hline Explicit & 1.0 & 2.550 & 2.550 & 0.000 \\
\hline Tacit & 1.0 & 20.000 & 20.000 & 0.000 \\
\hline Spiral & 1.0 & 22.550 & 22.550 & 0.000 \\
\hline
\end{tabular}

Now we examine the effect of halving, for instance, knowledge flow efficiency (i.e., $\mathrm{E}_{\mathrm{K}}=0.5$ ) in Table 7. As expected, K-Energy measurements remain unchanged across the three knowledge flow patterns, but their K-Work counterparts reflect half the previous (ideal) values. As expected likewise in this example, energy loss is identical to K-Work, as half the K-Energy is lost to inefficiency. These measurements match expectations and illustrate the considerable effect exerted by knowledge flow efficiency.
Table 7 Measurements $-\mathrm{E}_{\mathrm{K}}=0.5$

\begin{tabular}{|l|c|c|c|c|}
\hline Path & EK $_{\text {K }}$ & KE & KW & \multicolumn{1}{|c|}{ EL } \\
\hline Explicit & 0.5 & 2.550 & 1.275 & 1.275 \\
\hline Tacit & 0.5 & 20.000 & 10.000 & 10.000 \\
\hline Spiral & 0.5 & 22.550 & 11.275 & 11.275 \\
\hline
\end{tabular}

To increase insight into the three knowledge flow patterns, we solve to determine what comparative efficiency levels would be required for each knowledge flow to achieve the same K-Work level. From a management perspective, this helps to inform a decision regarding which knowledge flow pattern to adopt. Say, for example, that management establishes a K-Work target of $15,000 \mathrm{P}$ for the same 100 knowledge chunks and 10 people associated with the flow patterns above. Working backward from this target for each knowledge flow pattern, we calculate the efficiency level that would be required to hit such target. Results are reported in Table 8.

Table 8 Measurements - KW targets

\begin{tabular}{|l|c|c|c|r|}
\hline Path & $\mathbf{E}_{\mathbf{K}}$ & $\mathbf{K E}$ & $\mathbf{K W}$ & \multicolumn{1}{c|}{$\mathbf{E}_{\mathbf{L}}$} \\
\hline Explicit & $\mathbf{5 . 8 8}$ & 2.550 & 15.000 & $\mathbf{- 1 2 . 4 5 0}$ \\
\hline Tacit & 0.75 & 20.000 & 15.000 & 5.000 \\
\hline Spiral & 0.67 & 22.550 & 15.000 & 7.550 \\
\hline
\end{tabular}

For the Explicit Path we see immediately that hitting the K-Work target appears to be infeasible. Because the inherent K-Energy $(2550 \mathrm{P})$ is comparatively low, the knowledge flow would require an efficiency level of nearly six (5.88). Given our expectations of imperfect knowledge flow efficiency (i.e., $E_{K} \leq 1$ ), this implies that the organization would need to add over 12,000 Polanyis of K-Energy to the flow. This is reflected by the negative value for energy loss $(-12,450)$, which is infeasible. Given the K-Work target, management would clearly not choose the Explicit Path pattern for its desired knowledge flow.

Alternatively, knowledge flow efficiency levels for both the Tacit Path (0.75) and Spiral Model (0.67) are within the feasible range $[0,1]$, and management could pursue either flow pattern with the goal of hitting its established K-Work target. Regarding the choice between these two (and myriad others not described, delineated or measured here), management may favor the Spiral pattern, for it could reach the 15,000 P K-Work target at a lower knowledge flow efficiency level than required for the Tacit pattern (i.e., 0.67 vs. 0.75 ); in other words, the target would require lower efficiency (and hence be easier) to hit. However, management may favor the tacit pattern instead, seeking to hit the K-Work target with less K- 
Energy than associated with the Spiral pattern (i.e., 20,000 vs. 22,550 P).

Management could also look further to other measurements such as flow time for decision guidance. Recall from Table 5 that the measured value for Spiral Model flow time (71,200 s) exceeds that reported for the Tacit Path (55,100 s). This would likely lead management to favor the Tacit Path even further, for the knowledge would flow more quickly than through the Spiral pattern. This presumes of course that the organization is capable of attaining the requisite knowledge flow efficiency level (i.e., 0.75) associated with the Tacit Path.

Similarly, management could look to other measurements such as K-Power for decision guidance as well. Recall from Table 5 that K-Power of the Tacit Path (0.36) is slightly greater than that of the Spiral Model (0.32). This suggests that the greater K-Energy flowing through the Spiral does not quite compensate for the longer flow time. Such result offers still more support for the Tacit Path.

In either case, we see how management can utilize the knowledge flow efficiency measure, in addition to others such as flow time and K-Power, to support organization decision making. This represents a substantial step forward, and it opens up an exciting new connection between Knowledge Management and Decision Support.

\section{Conclusion}

Knowledge is key to competitive advantage, but it is inherently invisible, intangible and resistant to quantification, particularly when in dynamic motion. Recent research builds upon emerging knowledge measurement techniques and well-established knowledge flow theory to develop a system for measuring dynamic knowledge in the organization. Results from application to archetypical organization processes are highly consistent with extant theory, but they also lead us to question some theoretic concepts and correspondences.

In this article, we extend our system for measuring dynamic knowledge in the organization to specify the effects of knowledge flow efficiency. We illustrate how the knowledge flow efficiency measure $\left(E_{K}\right)$ can be used to compare a variety of organization flow patterns. We also demonstrate the considerable efficiency effect exerted on knowledge flows that experience energy losses.

By combining knowledge flow efficiency with other measures such as flow time and K-Power, we then elucidate a novel decision support capability, through which organization leaders and managers can utilize knowledge measures for decision making.
This represents a substantial step forward and opens up an exciting new connection between Knowledge Management and Decision Support.

Future research can work to understand how the extended system of knowledge equations can meld with and leverage the considerable Decision Support literature. Knowledge is clearly fundamental to decision making, hence $\mathrm{KM}$ is likely indispensable to decision support. Indeed, the field may benefit greatly by examining how measurements such as knowledge flow efficiency, flow time and K-Power can be used both in theory and by managers in practice.

Future research can work also to extend KM through dynamic knowledge visualization and measurement. We have illustrated how visualization and measurement can be applied to a few archetypical models from the literature. Yet a great many KM theories and models remain unexplored along these lines. A great many KM theories and models also remain conceptual and descriptive for the most part. Dynamic knowledge visualization and measurement offer novel potential to complement them with quantitative and prescriptive insights.

Future research can work further to measure the knowledge flows of operational organizations in the field. As such measurements accumulate, we may be able to establish an increasingly rich set of data for use in comparing different organizations, processes, technologies and knowledge flows on a quantitative basis. Perhaps we can even establish new sets of norms, benchmarks and like measures that can be utilized practically and productively by organization leaders and managers.

Despite these contributions and future research opportunities, it is important to emphasize that we recognize the limitations of analogic reasoning: In no way are we asserting that the dynamics of knowledge follow or mirror the dynamics of physical systems precisely. Every analogy breaks down when stretched too far, and even some of the most basic physical concepts may have little meaning in terms of dynamic knowledge, or vice versa. Notwithstanding such limitations, however, we gain insight from the deep understanding and mathematic representation of dynamic physical systems, which are adapted here to enable the measurement of dynamic knowledge.

Knowledge measurement remains a nascent research endeavor. Although the dynamic knowledge measurement system described in this article draws analogically from Physics, the study of dynamic knowledge systems is many centuries behind in terms of understanding with respect to dynamic physical systems. Even small, admittedly imprecise, analogic, 
theoretic steps such as ours can contribute much. We welcome others to contribute likewise.

\section{References}

[1] Ahn, J., \& Chang, S. (2004). Assessing the contribution of knowledge to business performance: The KP3 methodology. Decision Support Systems, 36(4), 403-416.

[2] Casakin, H. (2010). Visual analogy, visual displays, and the nature of design problems: The effect of expertise.

Environment and Planning B: Planning and Design, 37(1), 170-188.

[3] Cole, R. E. (1998). Introduction. California Management Review, 40(3), 15-21.

[4] Dierickx, I., \& Cool, K. (1989). Asset stock accumulation and sustainability of competitive advantage. Management Science, 35(12), 1504-1511.

[5] Drucker, P. F. (1995). Managing in a time of great change. New York: Truman Talley Books/Dutton.

[6] Gavetti, G., \& Rivkin, J. W. (2005). How strategists really think: Tapping the power of analogy. Harvard Business Review, 83(4), 54-63.

[7] Grant, R. M. (1996). Toward a knowledge based theory of the firm. Strategic Management Journal, 17, 109-122.

[8] Kolodner, J. L. (1994). Understanding creativity: A case-based approach. In: S. Wess S., K. Althoff, \& M. Richter (Eds.). Topics in Case-Based Reasoning. Berlin: Springer.

[9] Naim, M. M., Spiegler, V. M., Wikner, J., \& Towill, D. R. (2017). Identifying the causes of the bullwhip effect by exploiting control block diagram manipulation with analogical reasoning. European Journal of Operational Research, 263(1), 240-246.

[10] Nissen, M. E. (2002). An extended model of knowledge-flow dynamics. Communications of the Association for Information Systems, 8(18), 251-266.

[11] Nissen, M. E. (2006a). Dynamic knowledge patterns to inform design: A field study of knowledge stocks and flows in an extreme organization. Journal of Management Information Systems, 22(3), 225-263.

[12] Nissen, M. E. (2006b). Harnessing knowledge dynamics: Principled organizational knowing and learning. Hershey, PA: IRM Press.

[13] Nissen, M. E. (2014). Harnessing dynamic knowledge principles for competitive advantage in the technologydriven world. Hershey, PA: IGI Global.
[14] Nissen, M. E. (2017). Working toward a system for measuring dynamic knowledge. International Journal of Knowledge Management, 13(3), 1-19.

[15] Nissen, M. E. (2019). Applying a system for measuring dynamic knowledge: Reconsidering the Spiral Model. Proceedings Hawaii International Conference on System Sciences. Wailea, HI.

[16] Nonaka, I. (1994). A dynamic theory of organizational knowledge creation. Organization Science, 5(1), 14-37.

[17] Peterson, M. (1997). The use of analogies in developing outer space law. International Organization, 51(2), 245-274.

[18] Phye, G. D. (1997). Handbook of academic learning: Construction of knowledge. Burlington: Elsevier Science.

[19] Podolefsky, N. S., \& Finkelstein, N.D. (2007). Salience of representations and analogies in Physics. AIP Conference Proceedings, 951(1), 164-167.

[20] Polanyi, M. (1967). The tacit dimension. Garden City, N.Y.: Anchor Books.

[21] Preiss, K. (1999). Modelling of knowledge flows and their impact. Journal of Knowledge Management, 3(1), 3646.

[22] Simon, H. A. (1996). The sciences of the artificial (3rd. ed.). Cambridge, MA: The MIT Press.

[23] Spender, J. C. (1996). Making knowledge the basis of a dynamic theory of the firm. Strategic Management Journal, 17, 45-62.

[24] Sternberg, R. J. (1977). Intelligence, information processing, and analogical reasoning: The componential analysis of human abilities. Hillsdale, N.J.: Lawrence Erlbaum Associates.

[25] Sternberg, R. J., \& Rifkin, B. (1979). The development of analogical reasoning processes. Journal of Experimental Child Psychology, 27(2), 195-232.

[26] Szulanski, G. (2000). The process of knowledge transfer: A diachronic analysis of stickiness. Organizational Behavior and Human Decision Processes, 82(1), 9-27.

[27] Tsoukas, H. (1993). Analogical reasoning and knowledge generation in organization theory. Organization Studies, 14(3), 323-346. 